\title{
STOCHASTICALLY BOUNDED SOLUTIONS OF A LINEAR NONHOMOGENEOUS STOCHASTIC DIFFERENTIAL EQUATION
}

UDC 519.21

\author{
O. V. IL'CHENKO
}

\begin{abstract}
Conditions for the existence of a stochastically bounded solution of a linear nonhomogeneous stochastic differential equation are found in the paper. The stationary and periodic cases are considered.
\end{abstract}

The qualitative behavior of solutions of stochastic differential equations has recently been studied intensively. One problem that has been of permanent interest is to find conditions for the existence of stochastically bounded solutions, especially in view of the fact that stationary and periodic solutions possess this property.

A criterion for the existence of a unique stochastically bounded solution of a linear nonhomogeneous stochastic differential equation is obtained in this paper. It turns out that the existence of a unique stochastically bounded solution depends on whether a solution of the homogeneous equation is exponentially $p$-stable for some $p>0$ (see [3] for the definition). Note however that another condition (mean square stability) is common for problems of this kind (see [6, 7]). The main result of the paper is close to those well known in the case of deterministic systems (see, for example, [5]), and this indicates that it is definitive. We also show that the solution inherits properties of the coefficients of the equation if they are either stationary or periodic.

Consider the stochastic differential equation

$$
d x(t)=(b x(t)+f(t)) d t+\sum_{k=1}^{m}\left(\sigma_{k} x(t)+g_{k}(t)\right) d w_{k}(t),
$$

where $b$ and $\sigma_{k}$ are real constants; $f(t), t \in \mathbf{R}$, and $g_{k}(t), t \in \mathbf{R}$, are bounded continuous real functions; $\sup _{t \in \mathbf{R}}\left\{|f(t)|,\left|g_{k}(t)\right| ; k=1, \ldots, m\right\} \leq K<+\infty ; w_{k}(t)$ are onedimensional independent Wiener processes, $t \in \mathbf{R}, k=1, \ldots, m$.

A Wiener process $w(t), t \in \mathbf{R}$, is defined as a stochastic process with independent increments such that $w(0)=0$ and $w(t)-w(s)$ is a Gaussian random variable for all $s, t \in \mathbf{R}$ such that

$$
\mathrm{E}(w(t)-w(s))=0, \quad \mathbf{E}(w(t)-w(s))^{2}=|t-s| .
$$

In what follows we use the following flows of $\sigma$-fields:

$$
\begin{gathered}
\mathcal{F}_{t}=\sigma a\left\{w_{k}\left(s_{2}\right)-w_{k}\left(s_{1}\right): s_{1} \leq s_{2} \leq t, k=1, \ldots, m\right\}, \quad t \in \mathbf{R} ; \\
\mathcal{F}^{t}=\sigma a\left\{w_{k}\left(s_{2}\right)-w_{k}\left(s_{1}\right): t \leq s_{1} \leq s_{2}, k=1, \ldots, m\right\}, \quad t \in \mathbf{R} ; \\
\mathcal{F}_{t-v}^{t}=\sigma a\left\{w_{k}\left(s_{2}\right)-w_{k}\left(s_{1}\right): t-v \leq s_{1} \leq s_{2} \leq t, k=1, \ldots, m\right\}, \quad v \geq 0 .
\end{gathered}
$$

2000 Mathematics Subject Classification. Primary 60H10; Secondary 34F05. 
Definition. A solution $x(t), t \in \mathbf{R}$, of equation (1) is called stochastically bounded if

$$
\lim _{N \rightarrow+\infty} \sup _{t \in \mathbf{R}} \mathrm{P}\{|x(t)|>N\}=0 .
$$

Along with (11) we consider the corresponding homogeneous equation

$$
d h_{s}^{t}=b h_{s}^{t} d t+\sum_{k=1}^{m} \sigma_{k} h_{s}^{t} d w_{k}(t) .
$$

The latter equation for $s \leq t$ and $h_{s}^{s}=1$ has a solution

$$
h_{s}^{t}=\exp \left\{\gamma(t-s)+\sum_{k=1}^{m} \sigma_{k}\left[w_{k}(t)-w_{k}(s)\right]\right\}, \quad \gamma=b-2^{-1} \sum_{k=1}^{m} \sigma_{k}^{2}
$$

(see [2]). For an arbitrary $p \in \mathbf{R}$,

$$
\mathrm{E}\left(h_{s}^{t}\right)^{p}=\exp \left\{\left(\gamma+p 2^{-1} \sum_{k=1}^{m} \sigma_{k}^{2}\right)(t-s) p\right\} .
$$

The solution $x_{s}(t), s \leq t$, of equation (1) can be represented in the following form:

$$
\begin{aligned}
x_{s}(t)=h_{s}^{t}\left[x(s)+\int_{s}^{t}\left(h_{s}^{u}\right)^{-1}(f(u)\right. & \left.-\sum_{k=1}^{m} \sigma_{k} g_{k}(u)\right) d u \\
& \left.+\sum_{k=1}^{m} \int_{s}^{t}\left(h_{s}^{u}\right)^{-1} g_{k}(u) d w_{k}(u)\right]
\end{aligned}
$$

(see [2]). The behavior of $x_{s}(t)$ is determined by the integral terms in (4). Our current goal is to obtain properties of the integral terms.

Lemma 1. Assume that $\varphi(t)$ is a continuous function of $t \in \mathbf{R}$. Then the following reverse integration formula holds for stochastic integrals with $s \leq t$ :

$$
h_{s}^{t} \int_{s}^{t}\left(h_{s}^{u}\right)^{-1} \varphi(u) d w_{k}(u)=-\int_{t}^{s} h_{u}^{t} \varphi(u) d w_{k}(u)-\sigma_{k} \int_{t}^{s} h_{u}^{t} \varphi(u) d u .
$$

Proof of Lemma 1. Using properties of $h_{s}^{t}$ we have that, for $\Delta=(t-s) / n, u_{i}=s+i \Delta$, $\Delta w_{k}\left(u_{i}\right)=w_{k}\left(u_{i+1}\right)-w_{k}\left(u_{i}\right), i=0, \ldots, n-1$,

$$
\begin{aligned}
& h_{s}^{t} \int_{s}^{t}\left(h_{s}^{u}\right)^{-1} \varphi(u) d w_{k}(u)=\text { l. i.p. }_{n \rightarrow \infty} h_{s}^{t} \sum_{i=0}^{n-1}\left(h_{s}^{u_{i}}\right)^{-1} \varphi\left(u_{i}\right) \Delta w_{k}\left(u_{i}\right) \\
& \text { = l. i.p. } \sum_{n \rightarrow \infty}^{n-1} h_{u_{i}}^{t} \varphi\left(u_{i}\right) \Delta w_{k}\left(u_{i}\right) \\
& =\text { l. i.p. } \sum_{n \rightarrow \infty}^{n-1} h_{u_{i+1}}^{t}\left(1+b \Delta+\sum_{r=1}^{m} \sigma_{r} \Delta w_{r}\left(u_{i}\right)\right) \varphi\left(u_{i+1}\right) \Delta w_{k}\left(u_{i}\right) \\
& =- \text { l. i. p. } \sum_{n \rightarrow \infty}^{n-1} h_{u_{n-i}}^{t} \varphi\left(u_{n-i}\right)\left(w_{k}\left(u_{n-(i+1)}\right)-w_{k}\left(u_{n-i}\right)\right) \\
& -\sigma_{k} \text { l.i.p. } \sum_{n \rightarrow \infty}^{n-1} h_{i=0}^{t} \varphi\left(u_{n-i}\right)(-1)\left(\Delta w_{k}\left(u_{n-(i+1)}\right)\right)^{2} \\
& =-\int_{t}^{s} h_{u}^{t} \varphi(u) d w_{k}(u)-\sigma_{k} \int_{t}^{s} h_{u}^{t} \varphi(u) d u .
\end{aligned}
$$

Lemma 1 is proved. 
Taking (5) into account one can rewrite (41) in the form

$$
x_{s}(t)=h_{s}^{t} x(s)-\int_{t}^{s} h_{u}^{t} f(u) d u-\sum_{k=1}^{m} \int_{t}^{s} h_{u}^{t} g_{k}(u) d w_{k}(u), \quad s \leq t .
$$

Now we are going to find conditions for the convergence of the integrals in ([6) as $s \rightarrow-\infty$. Put $p_{0}=2|\gamma|\left(\sum_{k=1}^{m} \sigma_{k}^{2}\right)^{-1}$.

Lemma 2. Assume that

1) $\gamma<0$

2) $\varphi(t)$ is a continuous function such that $\sup _{t \in \mathbf{R}}|\varphi(t)| \leq K<+\infty$.

Then for $r=1,2$ and $0<p<p_{0}$ there are constants $T>0$ and $0<q<1$ such that

$$
\mathrm{P}\left\{\int_{t-T(n+1)}^{t-T n}\left(h_{u}^{t}|\varphi(u)|\right)^{r} d u>N^{r} 2^{-n}\right\} \leq L_{1} N^{-p} q^{n}
$$

for all $t \in \mathbf{R}, N>0$, and $n \in \mathbf{N}$.

Proof of Lemma 2. Applying (3) we obtain for $p>0$

$$
\begin{aligned}
& \mathrm{P}\left\{\int_{t-T(n+1)}^{t-T n}\left(h_{u}^{t}|\varphi(u)|\right)^{r} d u>N^{r} 2^{-n}\right\} \\
& \quad \leq \mathrm{P}\left\{\int_{0}^{T}\left(h_{t-T n-u}^{t}|\varphi(t-T n-u)|\right)^{r} d u>N^{r} 2^{-n}\right\} \\
& \quad \leq \mathrm{P}\left\{T K^{r} \sup _{0 \leq u \leq T}\left(h_{0}^{T n+u}\right)^{r}>N^{r} 2^{-n}\right\} \\
& \quad \leq\left(N^{-r} 2^{n} T K^{r}\right)^{p / r} \mathrm{E}\left(\sup _{0 \leq u \leq T} h_{T n}^{T n+u} h_{0}^{T n}\right)^{p} \\
& \quad=\left(N^{-r} 2^{n} T K^{r}\right)^{p / r} \mathrm{E}\left(\sup _{0 \leq u \leq T} h_{0}^{u}\right)^{p} \mathrm{E}\left(h_{0}^{T n}\right)^{p} \\
& \quad \leq L\left(T K^{r}\right)^{p} N^{-p} \exp \left\{\left(\gamma+p 2^{-1} \sum_{k=1}^{m} \sigma_{k}^{2}+T^{-1} \ln 2\right) T n p\right\},
\end{aligned}
$$

$L=\mathrm{E}\left(\sup _{0 \leq u \leq T} h_{0}^{u}\right)^{p}<+\infty$. Choose a sufficiently large number $T>0$ and put $L_{1}=L\left(T K^{r}\right)^{p}$ and $q=\exp \left\{\left(\gamma+p 2^{-1} \sum_{k=1}^{m} \sigma_{k}^{2}+T^{-1} \ln 2\right) T p\right\}$. Lemma 2 is proved.

Lemma 3. Let the assumptions of Lemma 2 hold. Then the limit

$$
\lim _{s \rightarrow-\infty} \int_{t}^{s} h_{u}^{t} \varphi(u) d u=\int_{t}^{-\infty} h_{u}^{t} \varphi(u) d u
$$

exists almost surely for all $t \in \mathbf{R}$.

Proof of Lemma 3. By the Borel-Cantelli lemma it is sufficient to prove that the series

$$
\sum_{n=1}^{\infty} \mathrm{P}\left\{\int_{t-T(n+1)}^{t-T n} h_{u}^{t}|\varphi(u)| d u>2^{-n}\right\}
$$

converges for some $T>0$. This result follows from Lemma 2 for $r=1$. Lemma 3 is proved.

Lemma 4. Let the assumptions of Lemma 2 hold. Then the limit

$$
\lim _{s \rightarrow-\infty} \int_{t}^{s} h_{u}^{t} \varphi(u) d w_{k}(u)=\int_{t}^{-\infty} h_{u}^{t} \varphi(u) d w_{k}(u)
$$

exists almost surely for all $t \in \mathbf{R}$. 
Proof of Lemma 4. Consider the process

$$
m_{k}^{t}(v)=\int_{t}^{t-v} h_{u}^{t} \varphi(u) d w_{k}(u)=\int_{0}^{v} h_{t-u}^{t} \varphi(t-u) d w_{k}^{t}(u), \quad v \geq 0
$$

$w_{k}^{t}(u)=w_{k}(t-u)-w_{k}(t), u \geq 0$. This is a martingale whose characteristic with respect to the flow $F_{t-v}^{t}, v \geq 0$, is given by

$$
\left\langle m_{k}^{t}\right\rangle(v)=-\int_{t}^{t-v}\left(h_{u}^{t} \varphi(u)\right)^{2} d u=\int_{0}^{v}\left(h_{t-u}^{t} \varphi(t-u)\right)^{2} d u .
$$

The proof of the existence of the limit

$$
\left\langle m_{k}^{t}\right\rangle(+\infty)=\lim _{v \rightarrow+\infty}\left\langle m_{k}^{t}\right\rangle(v)=\int_{0}^{+\infty}\left(h_{t-u}^{t} \varphi(t-u)\right)^{2} d u
$$

is similar to that of Lemma 3. This means that the square integrable martingale $m_{k}^{t}(v)$ is closed and therefore the limit (8) exists for this process. Lemma 4 is proved.

Theorem 1. In order that, for given continuous functions $f(t)$ and $g_{k}(t), k=1, \ldots, m$, bounded on the real axis, there exist a unique stochastically bounded solution $\tilde{x}(t), t \in \mathbf{R}$, of equation (11) it is necessary and sufficient that $\gamma \neq 0$. In the case of the existence, this solution is given by

$$
\tilde{x}(t)=\left\{\begin{aligned}
-\int_{t}^{-\infty} h_{u}^{t} f(u) d u-\sum_{k=1}^{m} \int_{t}^{-\infty} h_{u}^{t} g_{k}(u) d w_{k}(u), & \gamma<0 ; \\
-\int_{t}^{+\infty}\left(h_{t}^{u}\right)^{-1}\left(f(u)-\sum_{k=1}^{m} \sigma_{k} g_{k}(u)\right) d u & \\
-\sum_{k=1}^{m} \int_{t}^{+\infty}\left(h_{t}^{u}\right)^{-1} g_{k}(u) d w_{k}(u), & \gamma>0 .
\end{aligned}\right.
$$

Moreover

$$
\sup _{t \in \mathbf{R}} \mathrm{E}|\tilde{x}(t)|^{p}<+\infty
$$

for $0<p<p_{0}$.

Remark. The condition $\gamma \neq 0$ means that, given $0<p<p_{0}$, the solution $h_{s}^{t}$ of equation (2) is exponentially $p$-stable (if $\gamma<0$ ) or it is exponentially $p$-unstable (if $\gamma>0$ ). This follows immediately from (3). The number $p_{0}$ is called the stability index (see [1]).

Recall that a solution $h_{s}^{t}$ is exponentially $p$-stable (exponentially $p$-unstable), $p>0$, if there exists a constant $\lambda>0$ such that

$$
\mathrm{E}\left(h_{s}^{t}\right)^{p} \leq e^{-\lambda(t-s)}, \quad\left(\mathrm{E}\left(h_{s}^{t}\right)^{-p} \leq e^{-\lambda(t-s)}\right)
$$

(see [3]).

Proof. Sufficiency. We consider the cases of $\gamma<0$ and $\gamma>0$ separately.

1) Let $\gamma<0$. Put

$$
x_{-\infty}(t)=-\int_{t}^{-\infty} h_{u}^{t} f(u) d u-\sum_{k=1}^{m} \int_{t}^{-\infty} h_{u}^{t} g_{k}(u) d w_{k}(u), \quad t \in \mathbf{R} .
$$

The right-hand side of (10) exists for all $t$ by Lemmas 3 and 4 . Putting $x(s)=0$ in (6) and approaching the limit as $s \rightarrow-\infty$, we prove that $x_{-\infty}(t)$ is a solution of equation (11).

The process $x_{-\infty}(t)$ is measurable with respect to the flow $\mathcal{F}_{t}$.

Now we show that the process $x_{-\infty}(t), t \in \mathbf{R}$, is stochastically bounded. Since

$$
\begin{aligned}
\mathrm{P}\left\{\left|x_{-\infty}(t)\right|>N\right\} \leq & \mathrm{P}\left\{\left|\int_{t}^{-\infty} h_{u}^{t} f(u) d u\right|>N / m+1\right\} \\
& +\sum_{k=1}^{m} \mathrm{P}\left\{\left|\int_{t}^{-\infty} h_{u}^{t} g_{k}(u) d w_{k}(u)\right|>N / m+1\right\},
\end{aligned}
$$


it is sufficient to prove that every term in (10) is stochastically bounded. We have

$$
\mathrm{P}\left\{\left|\int_{t}^{-\infty}\left(h_{u}^{t} f(u)\right)^{r} d u\right|>N^{r}\right\} \leq L_{2} N^{-p}
$$

for $r=1,2,0<p<p_{0}$, and $L_{2}=L_{2}(p)<+\infty$, where the integral is defined for almost all trajectories. Indeed, by Lemma 2

$$
\begin{aligned}
\mathrm{P}\left\{\left|\int_{t}^{-\infty}\left(h_{u}^{t} f(u)\right)^{r} d u\right|>N^{r}\right\} & \leq \mathrm{P}\left\{\bigcup_{n=0}^{\infty}\left(\left|\int_{t-T(n+1)}^{t-T n}\left(h_{u}^{t} f(u)\right)^{r} d u\right|>N^{r} 2^{-(n+1)}\right)\right\} \\
& \leq \sum_{n=0}^{\infty} \mathrm{P}\left\{\int_{t-T(n+1)}^{t-T n}\left(h_{u}^{t}|f(u)|\right)^{r} d u>N^{r} 2^{-(n+1)}\right\} \\
& \leq 2^{p} L_{1}(1-q)^{-1} N^{-p} .
\end{aligned}
$$

We also have the following inequality for the distribution of stochastic integrals:

$$
\mathrm{P}\left\{\left|\int_{t}^{-\infty} h_{u}^{t} g_{k}(u) d w_{k}(u)\right|>N\right\} \leq L_{3} N^{-p}
$$

where $0<p<p_{0}$ and

$$
L_{3}=L_{3}(p)<+\infty .
$$

To check (13) we use the notation of Lemma 4 and put $\varphi(u)=g_{k}(u)$. Then

$$
\begin{aligned}
\mathrm{P}\left\{\left|\int_{t}^{-\infty} h_{u}^{t} g_{k}(u) d w_{k}(u)\right|>N\right\} & \leq \lim _{V \rightarrow+\infty} \mathrm{P}\left\{\sup _{0<v<V}\left|m_{k}^{t}(v)\right|>N\right\} \\
& \leq \lim _{V \rightarrow+\infty} N^{-p} \mathrm{E}\left(\sup _{0<v<V}\left|m_{k}^{t}(v)\right|\right)^{p} \\
& \leq \lim _{V \rightarrow+\infty} N^{-p} c_{p} \mathrm{E}\left\langle m_{k}^{t}\right\rangle^{p / 2}(V) \\
& \leq N^{-p} c_{p} \mathrm{E}\left(-\int_{t}^{-\infty}\left(h_{u}^{t} g_{k}(u)\right)^{2} d u\right)^{p / 2}
\end{aligned}
$$

for some $c_{p}<+\infty$ (see [4]). It remains to show that

$$
\mathrm{E}\left(-\int_{t}^{-\infty}\left(h_{u}^{t} g_{k}(u)\right)^{2} d u\right)^{p / 2}<+\infty, \quad t \in \mathbf{R}, 0<p<p_{0}
$$

We apply inequality (12) for $p+\delta, \delta=\left(p_{0}-p\right) / 2, f(u)=g_{k}(u)$, and $r=2$ :

$$
\begin{aligned}
\mathrm{E}\left(-\int_{t}^{-\infty}\left(h_{u}^{t} g_{k}(u)\right)^{2} d u\right)^{p / 2} & \leq \sum_{n=0}^{\infty} 2^{p(n+1)} \mathrm{P}\left\{4^{n}<-\int_{t}^{-\infty}\left(h_{u}^{t} g_{k}(u)\right)^{2} d u \leq 4^{n+1}\right\} \\
& \leq \sum_{n=0}^{\infty} 2^{p(n+1)} \mathrm{P}\left\{4^{n}<-\int_{t}^{-\infty}\left(h_{u}^{t} g_{k}(u)\right)^{2} d u\right\} \\
& \leq \sum_{n=0}^{\infty} 2^{p(n+1)} L_{2} 2^{-(p+\delta) n} \leq \sum_{n=0}^{\infty} L_{2} 2^{p} 2^{-\delta n}<+\infty .
\end{aligned}
$$

Now we prove relation (9). It follows from (11), (12) for $r=1$, and (13) that

$$
\mathrm{P}\left\{\left|x_{-\infty}(t)\right|>N\right\} \leq L_{3} N^{-p},
$$


$0<p<p_{0}, L_{3}=L_{3}(p)<+\infty$. Using (14) for $p+\delta$ and $\delta=\left(p_{0}-p\right) / 2$ we get

$$
\begin{aligned}
\mathrm{E}\left|x_{-\infty}(t)\right|^{p} & \leq \sum_{n=0}^{\infty} 2^{p(n+1)} \mathrm{P}\left\{2^{n}<\left|x_{-\infty}(t)\right| \leq 2^{n+1}\right\} \\
& \leq \sum_{n=0}^{\infty} 2^{p(n+1)} \mathrm{P}\left\{2^{n}<\left|x_{-\infty}(t)\right|\right\} \\
& \leq \sum_{n=0}^{\infty} 2^{p(n+1)} L_{3} 2^{-(p+\delta) n} \leq \sum_{n=0}^{\infty} L_{3} 2^{p} 2^{-\delta n}<+\infty .
\end{aligned}
$$

We have already proved that the process $x_{-\infty}(t), t \in \mathbf{R}$, is a stochastically bounded solution of equation (1), and inequality (9) holds for it if $\gamma<0$. A stochastically bounded solution is unique, since

$$
\mathrm{P}\left\{\lim _{s \rightarrow-\infty} h_{s}^{t}=+\infty\right\}=1 .
$$

2) Let $\gamma>0$. We show that in this case there is no stochastically bounded solutions of (1) that are measurable with respect to the flow $\mathcal{F}_{t}$. Consider relation (4). Since $-\gamma<0$, one can use the same method as that in Lemmas 3 and 4 and prove that the limit

$$
\varrho(s)=\lim _{t \rightarrow \infty}\left[\int_{s}^{t}\left(h_{t}^{u}\right)^{-1}\left(f(u)-\sum_{k=1}^{m} \sigma_{k} g_{k}(u)\right) d u+\sum_{k=1}^{m} \int_{s}^{t}\left(h_{t}^{u}\right)^{-1} g_{k}(u) d w_{k}(u)\right]
$$

exists almost surely for all $s \in \mathbf{R}$. It follows from $\mathrm{P}\left\{\lim _{t \rightarrow \infty} h_{s}^{t}=+\infty\right\}=1$ that if $x(s)$, $s \in \mathbf{R}$, is stochastically bounded, then $x(s)=\varrho(s)$ almost surely. This means that the random variable $x(s)$ is $\mathcal{F}^{s}$-measurable. To construct an $\mathcal{F}^{t}$-measurable stochastically bounded solution, we use a representation for the solution $x^{s}(t), t \leq s$, determined by its value $x(s)$ at the moment $s$. Applying formula (5) to equality (4) and interchanging $s$ and $t$ we get

$$
x^{s}(t)=\left(h_{t}^{s}\right)^{-1}\left[x(s)+\int_{s}^{t} h_{u}^{s} f(u) d u+\sum_{k=1}^{m} \int_{s}^{t} h_{u}^{s} g_{k}(u) d w_{k}(u)\right]
$$

for $t \leq s$. Taking (15) into account we rewrite (15) as follows:

$$
\begin{aligned}
x^{s}(t)= & \left(h_{t}^{s}\right)^{-1} x(s)-\int_{t}^{s}\left(h_{t}^{u}\right)^{-1}\left(f(u)-\sum_{k=1}^{m} \sigma_{k} g_{k}(u)\right) d u \\
& -\sum_{k=1}^{m} \int_{t}^{s}\left(h_{t}^{u}\right)^{-1} g_{k}(u) d w_{k}(u), \quad t \leq s .
\end{aligned}
$$

Put $x(s)=0$. Reasoning as in the proofs of Lemmas 3 and 4 we prove the existence of the limit as $s \rightarrow+\infty$. As a result we obtain a solution of equation (1),

$$
\begin{aligned}
x^{+\infty}(t)= & -\int_{t}^{+\infty}\left(h_{t}^{u}\right)^{-1}\left(f(u)-\sum_{k=1}^{m} \sigma_{k} g_{k}(u)\right) d u \\
& -\sum_{k=1}^{m} \int_{t}^{+\infty}\left(h_{t}^{u}\right)^{-1} g_{k}(u) d w_{k}(u) .
\end{aligned}
$$

The process $x^{+\infty}(t)$ is $\mathcal{F}^{t}$-measurable. Similarly to the case of $\gamma<0$, we check that the solution $x^{+\infty}(t), t \in \mathbf{R}$, is stochastically bounded and possesses the $p$ th moment for $0<p<p_{0}$. Since $\mathrm{P}\left\{\lim _{t \rightarrow-\infty}\left(h_{t}^{s}\right)^{-1}=+\infty\right\}=1$, the solution is unique. 
To complete the proof we put

$$
\tilde{x}(t)= \begin{cases}x_{-\infty}(t), & \gamma<0 \\ x^{+\infty}(t), & \gamma>0 .\end{cases}
$$

The necessity is proved by contradiction. Let $\gamma=0$. We show that there exist continuous and bounded functions $f(t)$ and $g_{k}(t), k=1, \ldots, m$, such that equation (1) has no stochastically bounded solution. Consider the equation

$$
d x(t)=(x(t)+1) d t+\sqrt{2} x(t) d w(t) .
$$

The solution of equation (17) for $t \geq s$ is of the form

$$
x_{s}(t)=\exp \{\sqrt{2} w(t)\}\left[x(s)+\int_{0}^{t} \exp \{-\sqrt{2} w(u)\} d u\right] .
$$

The total time spent by the trajectory of the Wiener process $-w(t)$ in the positive halfplane tends to $+\infty$ as $t \rightarrow+\infty$, whence for arbitrary $x(s)$

$$
\lim _{t \rightarrow+\infty}\left[x(s)+\int_{0}^{t} \exp \{-\sqrt{2} w(u)\} d u\right]=+\infty
$$

almost surely and therefore in probability. Since

$$
\mathrm{P}\{\exp \{\sqrt{2} w(t)\} \geq 1\}=1 / 2
$$

we conclude that there is no stochastically bounded solutions of equation (17). Therefore $\gamma \neq 0$. Theorem 1 is proved.

Theorem 2. Suppose the functions

$$
f(t)=f, \quad g_{k}(t)=g_{k}, \quad k=1, \ldots, m,
$$

in equation (1) do not depend on $t$. A unique stationary solution of equation (11) exists if and only if $\gamma \neq 0$.

Theorem 3. Suppose the functions

$$
f(t), \quad g_{k}(t), \quad k=1, \ldots, m,
$$

in equation (11) are continuous and periodic with period T. A unique T-periodic solution of equation (11) exists if and only if $\gamma \neq 0$.

Proof of Theorems 2 and 3. Stationary and periodic solutions of equation (1) are stochastically bounded. Thus, to prove Theorems 2 and 3, one must check the corresponding properties of the distributions of $x_{-\infty}(t)$ and $x^{+\infty}(t)$. This can be done in a standard way (see [8]). Theorems 2 and 3 are proved.

Acknowledgement. The author is grateful to A. A. Dorogovtsev for useful comments.

\section{BIBLIOGRAPHY}

1. L. Arnold and R. Khasminskiǔ, Stability index for nonlinear stochastic differential equations, Stochastic Analysis (M. Cranston and M. Pinsky, eds.), Proc. Sympos. Pure Math., vol 57, Amer. Math. Soc., Providence, RI, 1995, 543-552. MR 96e:60100

2. I. I. Gikhman and A. V. Skorokhod, Stochastic Differential Equations, "Naukova Dumka", Kiev, 1968; English transl., Springer-Verlag, Berlin, 1972. MR 41:7777; MR 49:11625

3. A. V. Skorokhod, Asymptotic Methods in the Theory of Stochastic Differential Equations, "Naukova Dumka", Kiev, 1987; English transl., Amer. Math. Soc., Providence, RI, 1989. MR 88m:60164 MR 90i:60038

4. N. Ikeda and S. Vatanabe, Stochastic Differential Equations and Diffusion Processes, NorthHolland, Amsterdam, 1981. MR 84b:60080 
5. Yu. L. Daletskiü and M. G. Krein, Stability of Solutions of Differential Equations in Banach Spaces, "Nauka", Moscow, 1970; English transl., Amer. Math. Soc., Providence, RI, 1974. MR 50:5125

6. A. Ya. Dorogovtsev, Periodic and Stationary Regimes of Infinite-Dimensional Deterministic and Stochastic Dynamic Systems, "Vyshcha Shkola", Kiev, 1992. (Russian) MR 94c:60097

7. A. Ichikawa, Bounded and Periodic Solutions of a Linear Stochastic Evolution Equations, Lect. Notes Math., vol. 1299, Springer-Verlag, 1988, 124-130. MR 89f:60061

8. R. Z. Khas'minskiŭ, Stability of Systems of Differential Equations under Random Perturbations of Their Parameters, "Nauka", Moscow, 1969; English transl., Sijthoff \& Noordhoff, Alphen aan Rijn, 1980. MR 41:3925; MR 82b:60064

Department of Mathematics, Mechanics and Mathematics Faculty, Kyiv National Taras Shevchenko University, Academician Glushkov Avenue 6, Kyiv 02022, Ukraine

E-mail address: avi@univ.kiev.ua

Received 17/JUN/2002

Translated by V. SEMENOV 